\title{
Innovative Variables to Increase the Analysis of Maintenance of Industrial Equipment
}

\author{
Manuel Vicente Castillo Guilarte and Jason Arturo Castillo Araujo \\ Department of System Engineering, National Experimental Polytechnic University UNEXPO, Caracas 1000, Venezuela
}

\begin{abstract}
In existing references, authors and companies have defined the maintenance variables over time; it is found that in some cases there are traditions, incorrect postulates or the inappropriate use of terminology, which leads to a misinterpretation of the results when maintenance management is analyzed. Starting from a documentary and deductive methodology, in this article, the analysis of some authors is presented and, from its origin, the initial variables that allow the construction of adequate algorithms for the maintenance function and to obtain the correct information related to it are re-defined. In a simple way with mathematical models, to then apply statistical models which allows, subsequently, calculating the maintenance information and achieving that professionals, professors and students are incorporated into maintenance analysis processes with a higher cognitive level, producing continuous information and comprehensive to analyze and investigate industrial equipment based on a basic history.
\end{abstract}

Key words: Maintenance variables, maintenance parameters, maintenance analysis.

\section{Introduction}

When a purchase order is generated for the acquisition of a device with its specifications; a start is established for the buyer or entrepreneur; previously there was a definition of what you want to buy from a project, the design of the industrial plant and the establishment of the different teams that make it up. In turn, the manufacturer builds the equipment with a series of variables, previous in a design and assembly that establishes the characteristics, specifications and operating conditions of preventive, corrective and major maintenance required [1], that is, in general, the initial or antecedent information. When the equipment starts to work, after the process of installation, testing, adjustment and commissioning, it becomes a source of data and information and, for this, it is necessary that the equipment is in normal operation according to the manufacturer and, adding the variables associated with the environmental conditions and quality of professionals involved, the team produces its own data,

Corresponding author: Manuel Vicente Castillo Guilarte, Ph.D., research fields: technology and innovation, reliability and maintainability system. E-mail: mvcg22@gmail.com. establishing an integration of facts, jobs and conditions in variables known as historical maintenance information associated with the team and then, you can compare, analyze, correct, modify, improve, conserve and control the source according to the values obtained and increase the precision of the analysis with more statistical variables.

There are authors [2], who state: "that they do not reflect any standard theory about the management of a safe maintenance program”. But it turns out that to make a planning and or computer program, it is necessary to know accurately the source of the data or its origin and the definition of the variables associated with the data, to later apply mathematical and statistical models, and log maintain a proper relationship between reality and the decision making corresponding to the team, minimizing and controlling errors; In this way, procedures can be proposed to analyze the source of data, which are transformed into information, program-codes and algorithm for decision making with quantified and controlled error.

In this article, the data source is the equipment subject to maintenance and, once in operation, at some point they will fail, and constitutes the reality of the 
equipment as a normal process, operates and fails over time. For purposes of standardizing this study it is important to clarify that the equipment is of continuous operation, however, the production may be discrete, which means that when it does not produce being available it is an administrative stop, in which preventive maintenance is done, but if to guarantee the goals the team must operate continuously, the system is unavailable, a failure occurs because of a preventive which is part of how a plant is designed and how it will be preventive, corrective and conservation maintenance. Much has been written in this subject and authors [1], defines the TBF (time between failure), other authors define the time of permanence (TP), operating time (OT), production time (PT) [3], time of operation (TO) [4] and questions arise: How do I interpret? How do I calculate it? How do I obtain time? What is it really?

This document is organized as follows: in Section 2 the objective of the work is stated, in Section 3, the generation of data of a team, in Section 4 it is established what historic information should be of the equipment, in the Section 5 basic maintenance information is established, in the Section 6 the basic information system of maintenance of a team is defined and, finally, in Section 7 the conclusions.

\section{Objective of the Work}

The objective of this article is the standardization and the logic of the data associated to the failures of a team or plants, establishing the direct and indirect variables, to analyze and obtain, later, the maintenance information; achieving that professionals can, with this knowledge, build the algorithms, mathematical models and statistical maintenance of a team or several teams, controlling errors.

\section{Generation of Date of a Team}

Based on the idea that the team is the source of information, it only manifests itself when it fails, being the failure of each team, when it happens it is random, because there is no way to know exactly when it will generate it and not it is subject to control; the fault is defined as a random data and is that which occurs when the equipment does not comply with the design conditions, does not satisfy the quality and/or quantity of the products or does not fit into the production line. As soon as the equipment is started or started up for the first time, it begins to produce and the first data is quantified, it works until a fault occurs, and so on, which generates points in time, is illustrated in Fig. 1.

Failure being a random variable, associated with it there is a before and after each failure. To reach a fault is because the equipment was operating and the fault broke this continuity and, after the failure the equipment does not work, that is, before the failure the equipment was in service and after the failure, out of service but, both cases, strictly depend on the fault and between each of them there will be the time between failure and failure, which is not valid from the beginning because there was no failure, this is known as the time between failures (TBF); after the fault, there is time out of service plus the next operation, which can be seen in Fig. 2, where there is a union in which the failure ends, this occurs once it is repaired and put in service and the equipment operates until the next failure.

Based on real numbers, bounded superiorly [5], each real number is a data and since, these data are successive in time, we define the variables for which a name must be established.

Before the fault the equipment is in use, operating, operating, producing a service, the variable selected before the fault is the operating time (OT), when the failure occurs the subsequent variable must be defined as damaged, failed, out of service, under repair, the variable to select will be time out of service (TOS) and the time that passes from one failure to the next failure will be defined as time between failure (TBF), which is conformed by the sum of time out of service TOS (i) and, the following operating time OT (i + 1); 


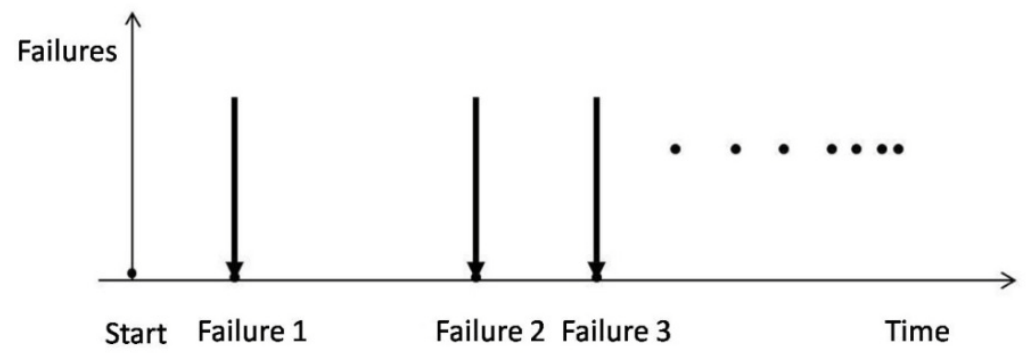

Fig. 1 Failure generating a point in time.

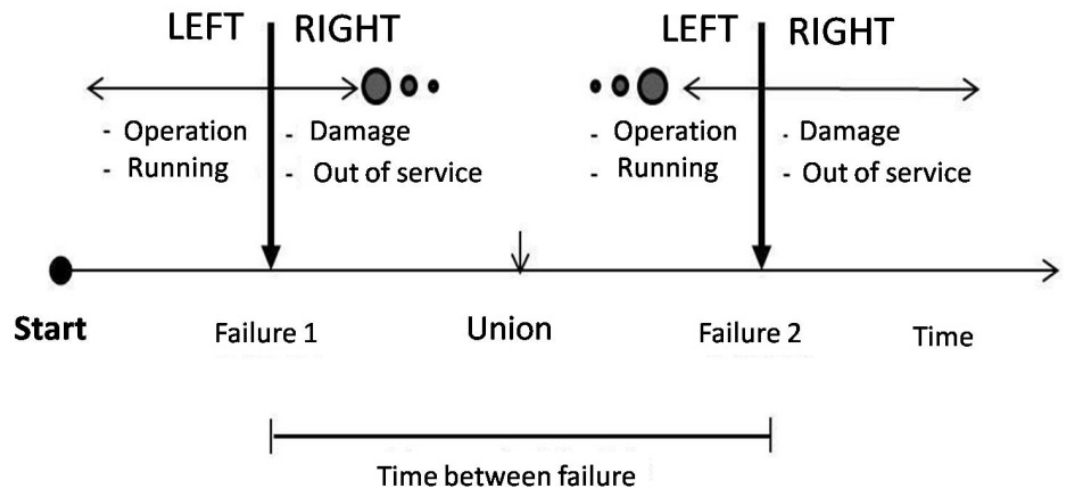

Fig. 2 Time between failures (TBF).

In this way, three random variables are established, a main one that is time between faults and linked to them and dependent on the random variables, operation time and time out of service.

It is important to highlight that the time out of service involves the administrative times of the failure (notification), logistic time (waiting for response and machinery), time of repair (action of corrective teams), delivery time and any other event that keep the equipment out of service. Fig. 3 illustrates how the data are presented in time as a consecutive process of change of state: it operates and fails, where, each point will be a calendar date being its arithmetic difference the elapsed time between a date and a successive one and, on the vertical side, binary logic of zero (0) is used as time out of service and one (1) as operating time.

In this case, each date will be defined by the calendar year, month, day, hour, minute, second, with an approximation, and then a base of the time that is usually the time is selected, forming a data structure, this it will always be approximate and with a limit depending on the unit of working time that is established, if it is the hour it will have fractions of minutes which leads to an estimated error by unit conversion.

The search of the data and its variables is to exert a greater control in the management of maintenance and this is achieved when it is forecast to obtain the approximate values of the possible events in the future. It is illustrated in Fig. 4 [6], although projections or forecasts are not part of this article.

Taking into account each failure, when this occurs as a random variable, it produces a point $y$, generates a quantity or time to the left as the operation time (OT) and to the right as time out of service (TOS), both being dependent random variables of the type of failures, but also a fourth random variable that is the magnitude of time of the sum of the operation time and out of service associated with each failure. This variable can be defined as the failure time (FT) and, in Fig. 5, its meaning is shown. In this way, in this article, the concept of random variables is extended from the failure, which the authors established in time as three 


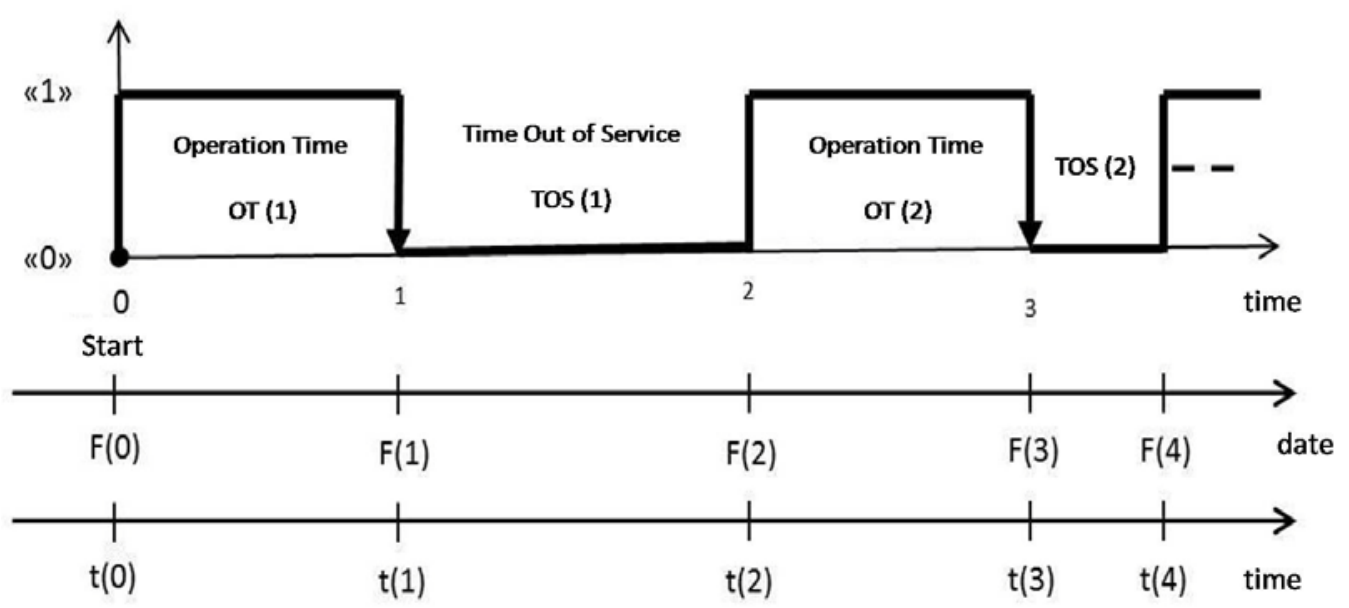

Fig. 3 Operation time and out of service time.

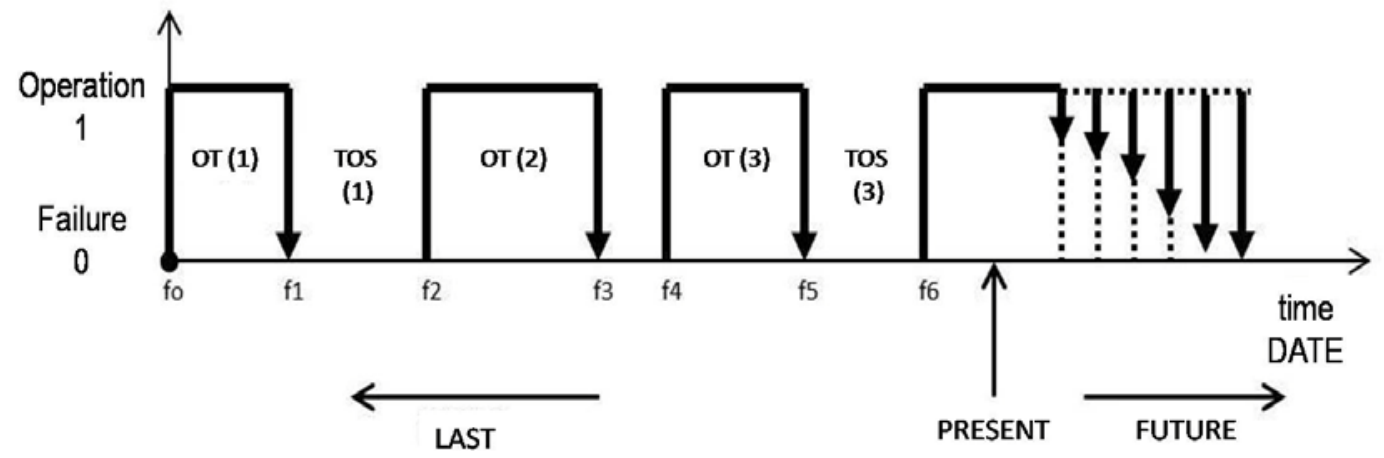

Fig. 4 Behavior of a team over time based on date.

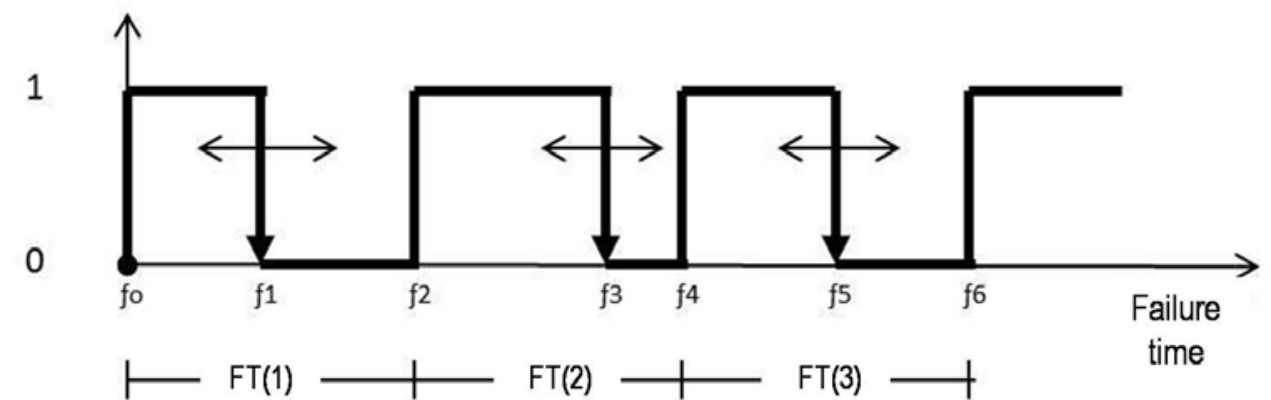

Fig. 5 Failure time (FT).

variables and, a fourth random variable is defined that is obtained from the failure of a equipment allows a greater analysis of the variables involved.

\section{Historical Information of the Equipment or Plant}

Each team must be inventoried with their identification code assignment [7] where all their information will be; being part of them the fault history of the equipment, which must have the starting date, start and dates of each failure and the dates of its restoration to service or operation, as presented in Table 1, based on Fig. 3, making it clear that it is active when a team's history is recorded, the work orders [8] are elaborated for each type of maintenance to obtain the fault history of the corrective maintenance. 
Table 1 History of equipment failures.

\begin{tabular}{llll}
\hline No. & Start date & Fail date & Cause \\
\hline 1 & $f(0)$ & $f(1)$ & $\mathrm{xxx}$ \\
2 & $f(2)$ & $\mathrm{f}(3)$ & $\mathrm{xxx}$ \\
3 & $f(4)$ & $f(5)$ & $\mathrm{xxx}$ \\
4 & $f(6)$ & $f(7)$ & $\mathrm{xxx}$ \\
$\ldots$ & $\ldots$ & $\ldots$ & $\ldots$ \\
\hline
\end{tabular}

From the history, the data are recorded as calendar date (year, month, day, hour and min.), which allows defining the values that are going to be associated with random variables and other mathematical variables, of which we have the following equations:

- Time between failure (TBF), in Eq. (1):

$$
\begin{gathered}
\mathrm{TBF}(\mathrm{i})=f(2 \mathrm{i}+1)-f(2 \mathrm{i}-1) \\
\mathrm{i}=1, \mathrm{n}
\end{gathered}
$$

- Operation time (OT), in Eq. (2):

$$
\begin{gathered}
\mathrm{OT}(\mathrm{i})=f(2 \mathrm{i}-1)-f(2 \mathrm{i}-2) \\
\mathrm{i}=1, \mathrm{n}
\end{gathered}
$$

- Time out of service (TOS), in Eq. (3):

$$
\begin{gathered}
\operatorname{TOS}(\mathrm{i})=f(2 \mathrm{i})-f(2 \mathrm{i}-2) \\
\mathrm{i}=1, \mathrm{n}
\end{gathered}
$$

- Failure time (FT), in Eq. (4):

$$
\mathrm{FT}(\mathrm{i})=f(2 \mathrm{i}-2)-f(2 \mathrm{i})
$$

- Middle operation time (MOT), in Eq. (5):

$$
\operatorname{MOT}(\mathrm{n})=\sum_{\mathrm{i}=1}^{\mathrm{n}} \mathrm{OT}(\mathrm{i}) / \mathrm{n}
$$

- Middle time out of service (MTOS), in Eq. (6):

$$
\operatorname{MTOS}(\mathrm{n})=\sum_{\mathrm{i}=1}^{\mathrm{n}} \operatorname{TOS}(\mathrm{i}) / \mathrm{n}
$$

- Middle time between failure (MTBF), in Eq. (7):

$$
\operatorname{MTBF}(\mathrm{n})=\sum_{\mathrm{i}=1}^{\mathrm{n}} \operatorname{TBF}(\mathrm{i}) / \mathrm{n}
$$

- Middle failure time (MFT), in Eq. (8):

$$
\operatorname{MFT}(\mathrm{n})=\sum_{\mathrm{i}=1}^{\mathrm{n}} \mathrm{FT}(\mathrm{i}) / \mathrm{n}
$$

- Total service time (TST), in Eq. (9):

$$
\operatorname{TST}(\mathrm{n})=\sum_{\mathrm{i}=1}^{\mathrm{n}}(\mathrm{OT}(\mathrm{i})+\operatorname{TOS}(\mathrm{i}))
$$

- Availability by failure (AF), in Eq.( 10):

$$
\mathrm{AF} \text { (i) = OT (i) / FT (i) }
$$

- Middle availability (MA), in Eq. (11):

$$
\operatorname{MA}(\mathrm{n})=\sum_{\mathrm{i}=1}^{\mathrm{n}} \mathrm{AF}(\mathrm{i}) / \mathrm{n}
$$

- Consumed useful life (CUL), in Eq. (12):

$$
\mathrm{CUL}=\sum_{\mathrm{i}=1}^{\mathrm{n}} \mathrm{FT}(\mathrm{i})
$$

Each failure that occurs, being a team or sub-system in operation generates four random variables, previously defined in time, the interaction between them, is illustrated in Fig. 6 and, is related to the total time of service (TST) which is the sum of the times of failures limited by the last event or the accumulated of the times that establishes the consumed useful life (CUL), which is the total time elapsed since commissioning independent of the fault; The operating time three OT (3) is not $100 \%$ realized and this value will only exist when the fault occurs five $\mathrm{F}$ (5) which is characterized as the F (n) future failure.

\section{Primary Information of the Analysis of the Data of Maintenance of a Team}

From each failure we define four random variables, before the failure the left side operating time (OT); to the right after the failure the time out of service (TOS); between failure and failure or point-to-point as time between failure (TBF) and failure time (FT) which is the sum of the times on the left and right of each fault. These times and the random effect are shown in Fig. 7, in which the four random variables are represented.

The behavior of the times between faults is studied, they are located in time, the consumed life is calculated and the failures occurred year after year in graphic form is illustrated in Fig. 8, with the faults occurring year after year and, it can be graphically approximated to the curve of the bathtub and also the fault rat (FR) can be calculated in hours that is the inverse is middle failure rate per year MFRA and, by approximation, it is also the inverse of the MOT, where the MTOS does not include the first OT and the MOT do not include the TOS. 


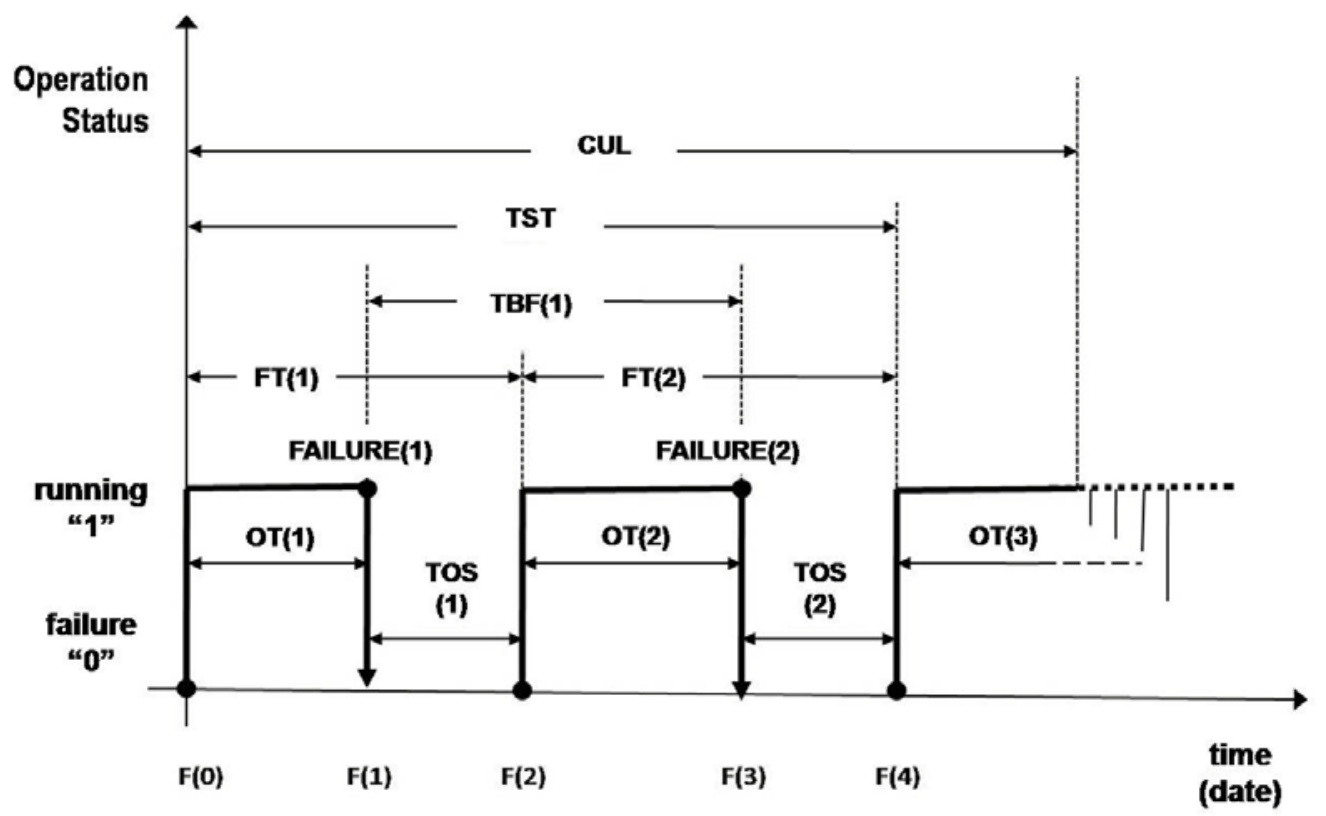

Fig. 6 Interaction of dates and times of a team.
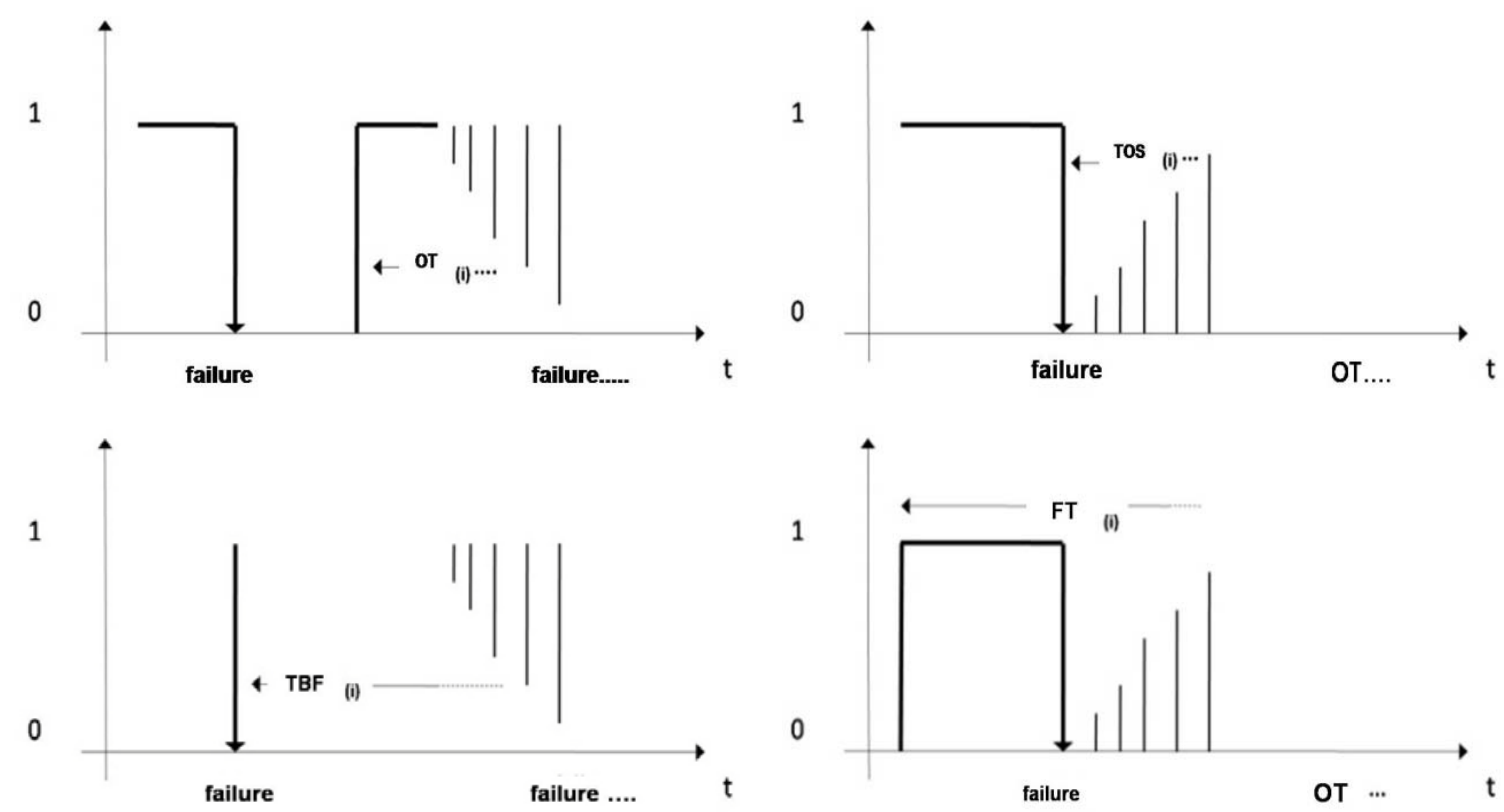

Fig. 7 Effects of the four random variables.

Mathematically it is divided between 8,760 hours of a year, between mean time between failures, which is represented in Eq. (13), establishing the middle failure rate per year (MFRY).

$\mathrm{MFRY}=\sum_{\mathrm{i}=1}^{\mathrm{n}}(\mathrm{TBF}(\mathrm{i}) / \mathrm{n})^{-1} * 8760$ horas/año (13)

- Consumed useful life (CUL), in Eq. (14):

$$
\mathrm{CUL}=\sum_{\mathrm{i}=1}^{\mathrm{n}} \mathrm{TBF}(\mathrm{i})+\mathrm{OT}(1)+\mathrm{OT}(\mathrm{n}) \quad(14)
$$

From Fig. 8 it can be plotted according to the number of failures per year, which allows us to approach the fault analysis, with the curve of the approximate bathtub; otherwise, more precision and greater complexity can be involved with statistical models.

With the inverse of the average operating times, the failures per hour will be obtained, which by multiplying them by 24 hrs/day, 720 hrs/month, 


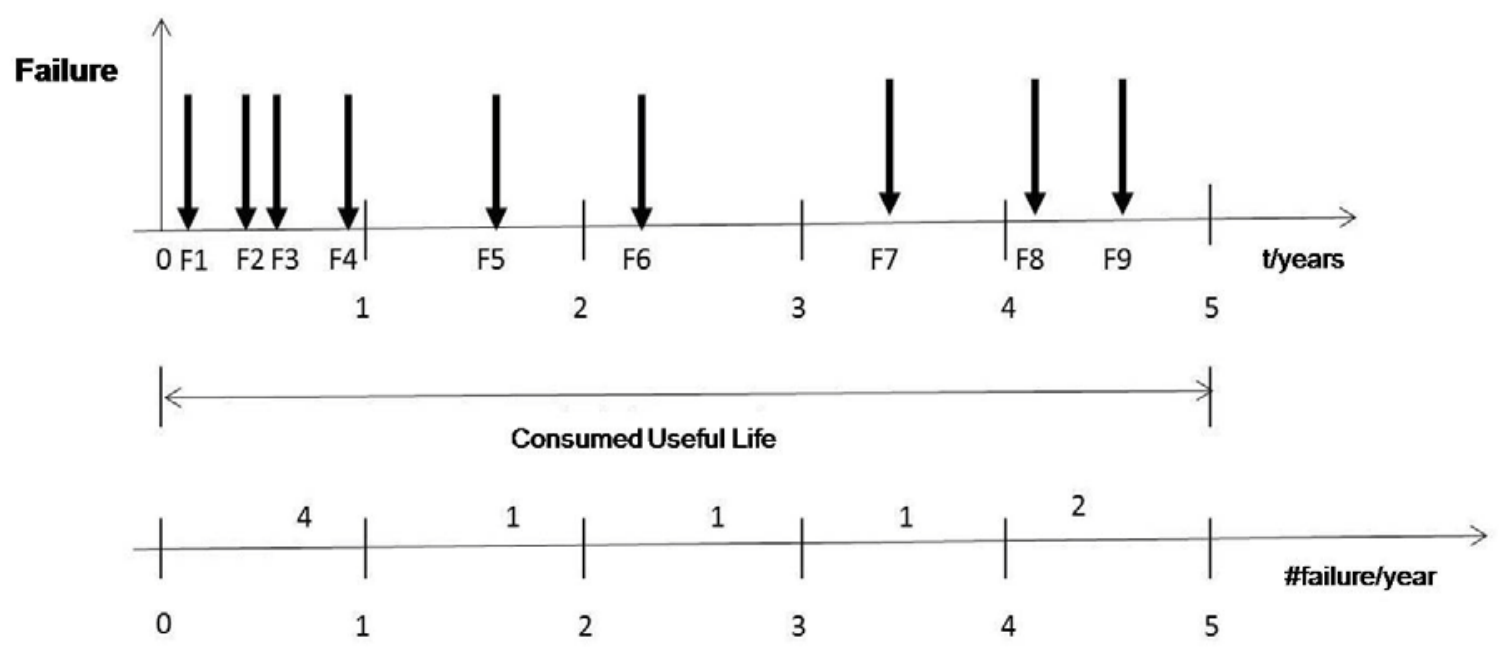

Fig. 8 Rat of failure in year intervals.

2,160 hrs/quarter, 4,320 hrs/semester and 8,760 hrs/year successively, results in the number of faults in each interval and, when plotting the curve, we obtain the basic behavior of the equipment over time and its average ratio of faults over time; the same can be done with exponential probability distributions or Weibull distributions which allows obtaining the curve with greater accuracy and forecasting and, the purpose is to determine how long the equipment works without failing and to determine when the next failure will most likely occur. When obtaining the inverse of the average times out of service when obtaining its inverse it is multiplied in a scale of hours, that is to say, 1 hour, 12 hrs, 24 hrs, 48 hrs, 72 hrs and $96 \mathrm{hrs}$, and the nearer one is the value of the equation to one (1) is the condition better.

Point or general availability is represented in Eq. (15), as a function of the average time of operation and out of service; it is also represented with the ratio of the average operating times between the useful life consumed.

$$
\mathrm{D}=\frac{\text { MOT }}{\mathrm{MOT}+\mathrm{MTOS}}=\frac{\mathrm{MOT}}{\mathrm{CUL}}
$$

\section{Variables for an Information System of Maintenance of a Team}

Starting from the equipment as a source of information, you must initially have the equipment history or obtain the information from the work orders and reconstruct the history only for the purpose of obtaining the data and establishing the variables, with the following sequence:

Initial start date $f(0)$

Dates associated with faults $f(\mathrm{i})$

Time between failures (TBF)

Operating time (OT)

Time out of service (TOS)

Failure time (FT)

Middle operating time (MOT)

Middle time out of service (MTOS)

Middle time between failure (MTBF)

Middle failure time (MFT)

Total service time (TST)

Availability by failure (AF)

Middle availability (MA)

Consumed useful life (CUL)

Rat of failure per hour (RF)

Middle rat failure per year (MFRY)

Availability (A)

\section{Conclusions}

The conclusions of the work are as follows:

(1) The presented development is a procedure that allows any professional to apply the logical sequence 
proposed and can develop the algorithm and program to obtain basic information of the maintenance parameters as a first approximation.

(2) From the development of this procedure, it is possible to deepen in statistical models that allow obtaining more maintenance information to improve decision making.

(3) This procedure manages and controls the source, data and maintenance information of a device.

(4) Since the equipment failures are a random variable in a point, four random variables are generated and configured, which allows studying the probability of failure, reliability, maintainability and availability.

(5) Once the maintenance parameters are determined, other variables associated with the equipment can also be calculated, such as: evaluation of the guarantee, quality, global management and others; each user will create their own maintenance information systems according to their needs.

\section{References}

[1] 1969. "Concepts of Optimization of the Maintenance." The Howard Finley Corporation Manual, USA, 30.

[2] Nava, J. D. 2012. Theory of Maintenance Reliability. Venezuela: Publications ULA.

[3] Leal, S., and Zambrano, S. 2013. Basic Basics of Maintenance, edited by Feunet, Venezuela, 31.

[4] Virtanen, S. 1998. "Reliability in Product Design-Specification of Dependability, Requirements." In Proceedings of the IEEE Reliability and Maintainability Symposium, 82-8.

[5] Iribarren, I. 2006. Introduction to the Theory of Measure. Venezuela: CDCH-UCV, 21.

[6] Araujo, J. C. 2015. "Process of Dynamic Teaching in Subjects of Maintenance at Undergraduate Level.” USB, Venezuela, 18.

[7] Guilarte, M. C. 1982. "Automated Model in Real Time to Obtain the Statistical Maintenance Parameters.” Master thesis, UCV, Venezuela, 26.

[8] Ángeles, R. G. 2009. Mantenimiento Industrial, edited by Trillas, México, 24. 